\title{
Steroids for All
}

\author{
Alexandra McKinzie ${ }^{1}$, Ziyi Yang ${ }^{2}$, Joanne Daggy², Robert Tepper ${ }^{3}$, Sarah Quinney ${ }^{1}$, Eli \\ Rhoads $^{3}$, Laura Haneline ${ }^{4}$, David M. Haas ${ }^{1}$ \\ ${ }^{1}$ Indiana University School of Medicine Department of Obstetrics and Gynecology; ${ }^{2}$ Indiana \\ University School of Medicine Department of Biostatistics, ${ }^{3}$ Indiana University School of \\ Medicine Division of Pediatric Pulmonology, ${ }^{4}$ Indiana University School of Medicine Division of \\ Neonatology
}

Background: Due to difficulties estimating the risk of preterm labor, many women diagnosed with threatened preterm delivery and given antenatal corticosteroids to improve neonatal outcomes do not deliver until term. Our objective was to compare the short-term outcomes of infants born at term to women who received betamethasone (BMZ) for threatened preterm labor to infants who were not exposed to BMZ in utero.

Methods: We performed a retrospective cohort study of infants born at or after 37 weeks' gestational age (GA) to mothers diagnosed with threatened preterm labor during pregnancy. Controlling for covariates, the primary neonatal outcomes of interest, including transient tachypnea of the newborn (TTN), neonatal intensive care unit (NICU) admission, and birthweight, were evaluated for their association with BMZ exposure.

Results: Of 5330 women, 1459 (27.5\%) women received $\mathrm{BMZ}$ at a mean GA of $32.2 \pm 3.3$ weeks. The mean age of women was $27 \pm 5.9$ years-old and the mean GA at delivery was $38.9 \pm 1.1$ weeks. Women receiving $B M Z$ had higher rates of maternal comorbidities $(P<0.001$ for diabetes, asthma, and hypertensive disorder) and were more likely to self-identify as white $(P=0.022)$. BMZ-exposed neonates had lower birth weights and increased rates of oxygen usage, TTN, hyperbilirubinemia, hypoglycemia, and NICU admission rates (all P-values <0.05). Controlling for maternal characteristics and GA at delivery, BMZ exposure was not significantly associated with diagnosis of TTN [OR $1.09(95 \% \mathrm{Cl} 0.80-1.50)]$, though it was associated with more NICU admissions [1.49 (1.19-1.86)] and lower birthweight by 91.5 (-122.3 to -60.6) grams.

Conclusions: Compared to women evaluated for preterm labor that did not receive BMZ, women receiving BMZ had infants with higher rates of NICU admission and lower birthweights, though the rate of TTN diagnosis was similar between the two groups. While the benefits of $\mathrm{BMZ}$ to infants born preterm are clear, there may be negative impacts for infants delivered at term. 